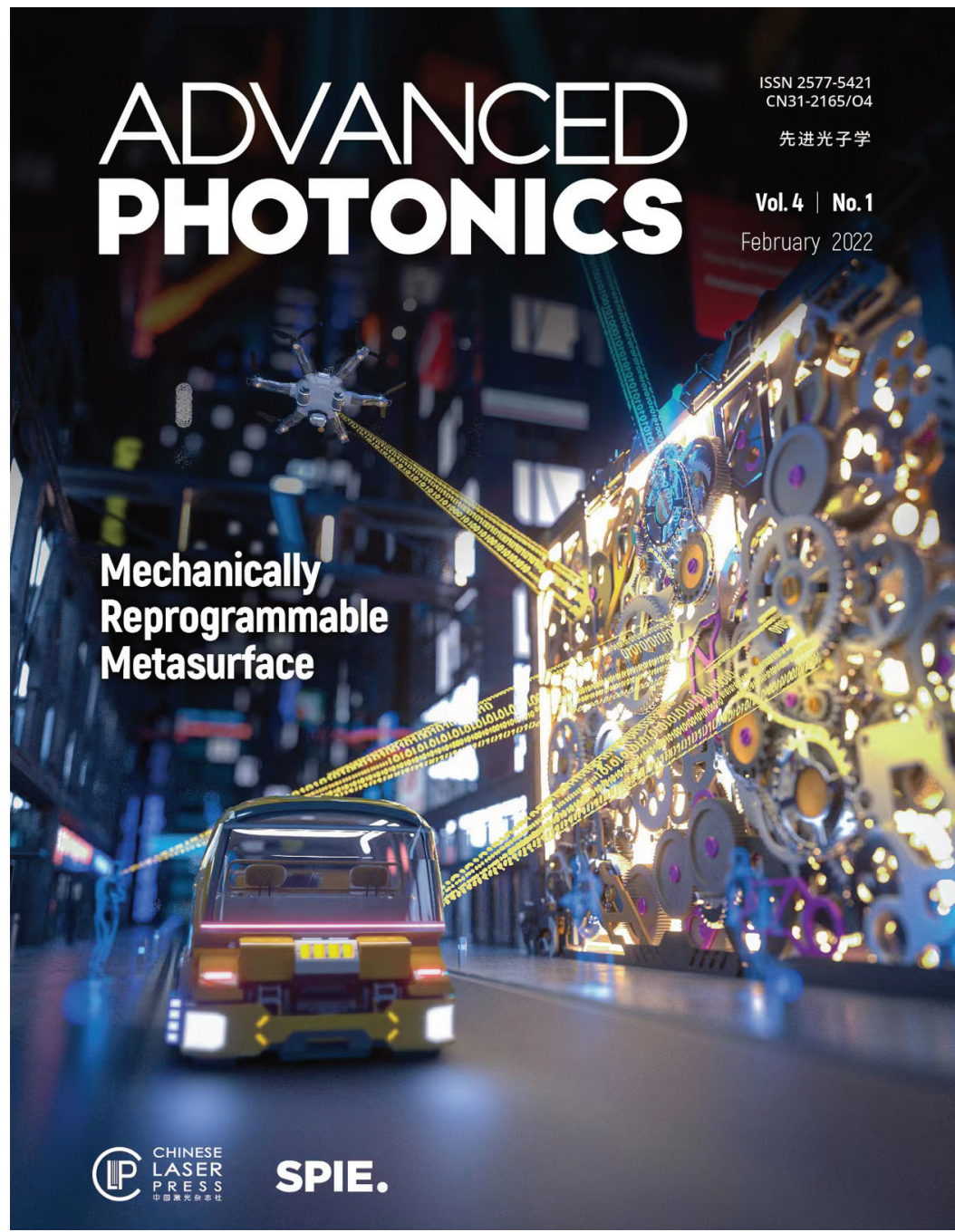

\title{
About the cover: Advanced Photonics Volume 4, Issue 1
}

Metasurfaces enable the realization of several optical functionalities over an ultrathin platform, fostering the exciting field of flat optics. Traditional metasurfaces are achieved by arranging a layout of static meta-atoms to imprint a desired operation on the impinging wavefront, but their functionality cannot be altered. Reconfigurability and programmability of metasurfaces are the next important step to broaden their impact, adding customized on-demand functionality in which each meta-atom can be individually reprogrammed. The image on the cover for Advanced
Photonics Volume 4 Issue 1 illustrates a reconfigurable intelligent surface that operates at microwave frequencies and uses a robust mechanical control method to flexibly determine the rotation angle of each meta-atom. The image is based on original research presented in the article "Mechanically reprogrammable Pancharatnam-Berry metasurface for microwaves," by Quan Xu, Xiaoqiang Su, Xueqian Zhang, Lijuan Dong, Lifeng Liu, Yunlong Shi, Qiu Wang, Ming Kang, Andrea Alù, Shuang Zhang, Jiaguang Han, and Weili Zhang. 